\title{
Can the Two-Time Interpretation of Quantum Mechanics Solve the Measurement Problem?
}

\author{
Katie Robertson \\ February 2017
}

Forthcoming in Studies in History and Philosophy of Science Part B - Studies in History and Philosophy of Modern Physics

\begin{abstract}
Over many years, Aharonov and co-authors have proposed a new interpretation of quantum mechanics: the two-time interpretation. This interpretation assigns two wavefunctions to a system, one of which propagates forwards in time and the other backwards. In this paper, I argue that this interpretation does not solve the measurement problem. In addition, I argue that it is neither necessary nor sufficient to attribute causal power to the backwards-evolving wavefunction $\langle\Phi|$ and thus its existence should be denied, contra the two-time interpretation. Finally, I follow Vaidman in giving an epistemological reading of $\langle\Phi|$.
\end{abstract}




\section{Introduction}

It is uncontroversial to say that there is no (global) consensus as to which interpretation of quantum mechanics should be adopted. In this paper, I will evaluate a new interpretation proposed by Aharonov, Gruss [2], Cohen and Landsberger [9] called the Two-Time Interpretation (TTI) ${ }^{1}$, which claims to be local, deterministic and to predict and explain novel phenomena. I will concentrate on whether this interpretation solves the measurement problem: since a prerequisite for joining the panoply of interpretations of quantum mechanics (QM) is to solve the measurement problem. I will argue that it does not.

The measurement problem arises as follows. When a measuring device ${ }^{2}$ measures a system initially in the state $\left|\Psi_{S}\right\rangle=\alpha\left|\uparrow_{x}\right\rangle+\beta\left|\downarrow_{x}\right\rangle$, the joint state of the system and apparatus evolves, by linearity, to

$$
\left.\left|\Phi_{S+A}\right\rangle=\alpha\left|\uparrow_{x}\right\rangle_{S}|u p\rangle_{A}+\beta\left|\downarrow_{x}\right\rangle_{S} \mid \text { down }\right\rangle_{A}
$$

The macroscopic measuring device is now entangled with the system and the composite system is in a superposition with respect to the product state basis. However, we never experience macroscopic superpositions, such as measuring devices pointing at both 'up' and 'down'. Rather, the measuring device will appear to display a single definite outcome.

In practice, in order to get empirical content from the formalism we apply a set of rules for measurement (sometimes called 'the measurement algorithm' [43]): write the state in the basis of the measured quantity/observable, and the pointer, $\alpha_{i}\left|a_{i}\right\rangle\left|A_{i}\right\rangle$, and then interpret $\left|\alpha_{1}\right|^{2}$ as the probability $(\operatorname{Pr})$ of finding state $\left|a_{1}\right\rangle$. In the case above, the measuring device is either found in the state $|u p\rangle$ with $\operatorname{Pr}=|\alpha|^{2}$ or $\mid$ down $\rangle$ with $\operatorname{Pr}=|\beta|^{2}$. Thus one way of describing the measurement problem is to say that this algorithm lacks a justification and 'to solve the measurement problem we need to give a well-formulated theory which explains the success of the measurement algorithm' [42].

In this paper I evaluate whether the TTI can solve this measurement problem. As the TTI arises from a particular reading of the two-state vector formalism (TSVF), I will first, in section 2, discuss the TSVF. In section 3, I outline the key tenets of the TTI as well as its proposed solution to the measurement problem.

\footnotetext{
${ }^{1}$ The two papers cited explicitly expound the TTI, but other papers such as [3] and [4] hint at it, since - as will become apparent shortly - the TTI naturally arises out of a wider research programme.

${ }^{2}$ As usual, we model a measurement by a composite unitary operator $U$ that takes the measuring device to one of several distinguishable (orthogonal) states depending on the system's state. Thus, a measuring apparatus that measures spin-x behaves as follows:
}

$$
\begin{gathered}
\left.U\left|\uparrow_{x}\right\rangle_{S} \mid \text { ready }\right\rangle_{A} \rightarrow\left|\uparrow_{x}\right\rangle_{S}|u p\rangle_{A} \\
\left.\left.U\left|\downarrow_{x}\right\rangle_{S} \mid \text { ready }\right\rangle_{A} \rightarrow\left|\downarrow_{x}\right\rangle_{S} \mid \text { down }\right\rangle_{A} .
\end{gathered}
$$


Section 4 first investigates whether the TTI explains the success of the measurement algorithm, and then whether it is well-formulated. In section 5, I argue that there are further problems facing this interpretation, and in section 6 I advocate an alternative reading of the situation.

\section{What is the two-state vector formalism?}

The two-state vector formalism (TVSF) $[4,1]$ alters the traditional formalism of QM by assigning an additional state vector $\langle\Phi|$ to a system, as well as the usual state vector, $|\Psi\rangle$. The latter state vector $\left|\Psi\left(t_{0}\right)\right\rangle$ unitarily evolves according to the Schrödinger equation, under $U$. However, the state $\left\langle\Phi\left(t_{1}\right)\right|$ evolves unitarily backwards in time under $U^{\dagger}$ and thus is called the 'backwards-evolving state vector'. ${ }^{3}$ Combined they form the two-state vector,

$$
\langle\Phi|| \Psi\rangle
$$

which also evolves unitarily. The philosophical motivation behind TSVF is to remove the time asymmetry implicit in the standard QM formalism, which Aharonov et al. [11] see as originating in the usual conception of state, rather than being inherent in QM. Using final and initial conditions is more time-symmetric than just initial conditions.

The TSVF is not an interpretation of QM: rather it is a mathematical formalism that applies to pre- and postselected (PPS) ensembles. A preselected ensemble is a prepared ensemble: all the systems gave the same outcome of a particular measurement at $t_{0}$. For example, it might be specified that the electrons in a Stern-Gerlach experiment are all initially $|\uparrow\rangle_{z}$. A postselected ensemble is defined analogously: e.g. those systems that gave the value $a_{i}$ for observable $A$ at time $t_{1}\left(>t_{0}\right)$.

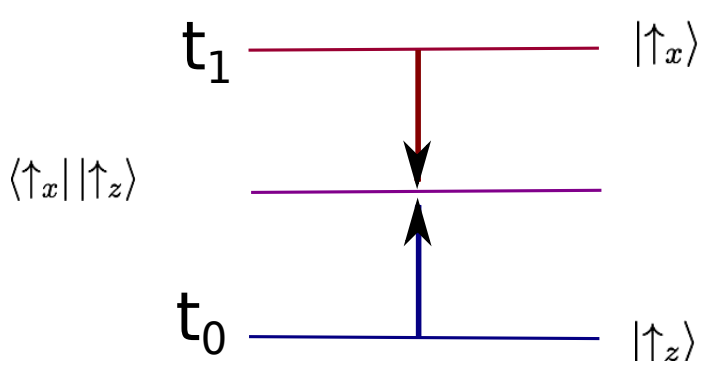

Figure 1: Preselection at $t_{0}: S_{z}=+\frac{1}{2}$. Postselection at $t_{1}: S_{x}=+\frac{1}{2}$.

Postselection can be viewed as specifying a final condition for the system just as preparation/preselection specifies an initial condition. A PPS system (a member of a PPS ensemble) therefore has two boundary conditions: a final as well as an

\footnotetext{
${ }^{3}$ In what follows, for brevity $\langle\Phi|$ denotes the generic backwards-evolving state.
} 


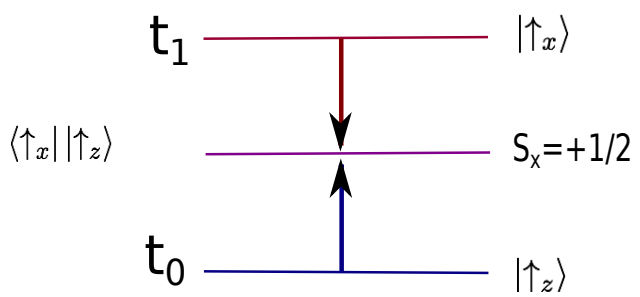

Figure 2: An intermediate measurement of $S_{x}$ in the period $t_{0}<t<t_{1}$ finds the eigenvalue $+\frac{1}{2}$ with $\operatorname{Pr}=1$.

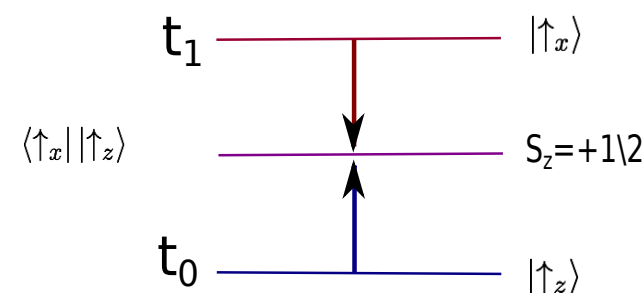

Figure 3: An intermediate measurement of $S_{z}$ in the period $t_{0}<t<t_{1}$ finds the eigenvalue $+\frac{1}{2}$ with $\operatorname{Pr}=1$.

initial condition. ${ }^{4}$ These boundary conditions (and therefore the two-state vector) are defined by measurement outcomes, as shown in Figure 1. We envisage that measurements are made on the system in the period $t_{0}<t<t_{1}$, as shown in Figures 2 and 3. Note how the situation differs from classical mechanics: classically, if we know the initial condition and the dynamics (the Hamiltonian) then the information contained in the final condition is redundant. However, in QM there is, for some measurements, no way even in principle to predict the result. Thus, specifying a final condition gives us more information about the system than just an initial condition.

Next, a method of determining the probabilities of outcomes of different measurements on PPS systems is needed. This is given by the Aharonov, Bergmann and Lebowitz (ABL) rule [1] which tells us, given an initial state $|a\rangle$ and a final state $|b\rangle$, the probability that an intermediate projective measurement of the non-degenerate operator $C$ yields eigenvalue $c_{i}$. We begin with the formula

$$
\mathcal{P}\left(c_{i} \mid a, b\right)=\frac{\mathcal{P}\left(b \mid c_{i}\right) \mathcal{P}\left(c_{i} \mid a\right)}{\Sigma_{j} \mathcal{P}\left(b \mid c_{j}\right) \mathcal{P}\left(c_{j} \mid a\right)} .
$$

Note that this expression is derived from the probability calculus: the Hamiltonian is set to zero and the only physical assumption is that $c_{i}$ screens off $b$ from $a$, i.e. the intermediate measurement is projective. Using $\operatorname{Pr}(g \mid f)=|\langle g \mid f\rangle|^{2}$, we find:

$$
\mathcal{P}\left(c_{i} \mid a, b\right)=\frac{\left|\left\langle b \mid c_{i}\right\rangle\left\langle c_{i} \mid a\right\rangle\right|^{2}}{\Sigma_{j}\left|\left\langle b \mid c_{j}\right\rangle\left\langle c_{j} \mid a\right\rangle\right|^{2}} .
$$

In the case of non-trivial time evolution, the $\mathrm{ABL}$ rule becomes:

$$
\mathcal{P}\left(c_{i} \mid a, b\right)=\frac{\left|\left\langle b\left|U\left(t_{b}, t\right)\right| c_{i}\right\rangle\left\langle c_{i}\left|U\left(t, t_{a}\right)\right| a\right\rangle\right|^{2}}{\Sigma_{j}\left|\left\langle b\left|U\left(t_{b}, t\right)\right| c_{j}\right\rangle\left\langle c_{j}\left|U\left(t, t_{a}\right)\right| a\right\rangle\right|^{2}} .
$$

For the PPS system shown in Figures 1-3, the ABL rule gives $\operatorname{Pr}=1$ for an intermediate result of $S_{z}=+\frac{1}{2}$ and likewise for $S_{x}=+\frac{1}{2}$. For $S_{y}=+\frac{1}{2}$, $\operatorname{Pr}=\frac{1}{2}$. The ABL rule is time-symmetric in the sense that, if the initial and final states are exchanged, then provided the Hamiltonian is time-reversal invariant,

\footnotetext{
${ }^{4}$ In practice, measurements on postselected ensembles involve doing a measurement on the whole preselected ensemble, then performing a selective measurement and discarding the results for systems that do not pass the postselection.
} 
the probabilities do not change. Note that for convenience the self-Hamiltonian (i.e. the Hamiltonian of the measured system) is often set to zero by advocates of this programme (for example, [9, p.138]), a practice which I follow in this paper. Gell-Mann and Hartle refer to the ABL rule as a "time-neutral formulation of quantum mechanics" [25, p.7].

Does TSVF have any advantage over the standard formalism? According to proponents of the TSVF, certain features of QM that would otherwise be opaque, are brought to light through the prism of the TSVF. For instance, discussing the path of a quantum particle through an interferometer is a fraught task: did the particle pass through both slits? Can we, or should, we assign a trajectory? In this connection, Vaidman suggests that the TSVF is illuminating: in particular, it is the right formalism for describing the past of a quantum particle [40, 19]. Additionally, the proponents of TSVF claim that the novel notion of 'weak values' naturally arise within this framework. The basic idea is that not all measurements are of the strong projective type assumed in the ABL rule (and in all the traditional treatments stemming from von Neumann 1932, ch.5-6): it is possible to weaken the interaction between the measuring device and system to such an extent that the measurement effectively no longer disturbs the system, which thus remains in its initial state. However, the results of such a 'weak' measurement contain such a large uncertainty in the pointer variable that an individual measurement will often reveal very little about the system. In TVSF, the 'weak value' of an observable $O$ on a system preselected as $\left|\Psi_{i}\right\rangle$ and postselected as $\left|\Psi_{f}\right\rangle$ is defined as

$$
O_{w}=\frac{\left\langle\Phi_{f}|O| \Psi_{i}\right\rangle}{\left\langle\Phi_{f} \mid \Psi_{i}\right\rangle}
$$

where $\left|\Psi_{i}\right\rangle$ and $\left|\Phi_{f}\right\rangle$ can be any two states provided $\left\langle\Phi_{f} \mid \Psi_{i}\right\rangle \neq 0$. Thus defined, weak values can be very counterintuitive; the weak value of a spin component of a spin- $\frac{1}{2}$ particle was measured to be 100 [6]. Unsurprisingly, the interpretation of weak values is controversial; in particular, it is disputed whether they represent a weaker feature of the system, or are complicated interference phenomena in the measuring device $[20,34]$. But according to Aharonov and Vaidman, these novel phenomena (weak values) are "very difficult to explain in the framework of standard quantum mechanics": and yet TVSF does explain them, while giving all the same empirical predictions as orthodox QM [4, p.3].

In the standard formalism, probabilities of measurement outcomes are given by the Born rule applied to preselected systems. This is formally recovered from the probabilities given by the ABL rule applied to PPS systems by assuming that nothing is known about the backwards-evolving state $\langle b|$. Summing over a complete orthonormal basis - which amounts to not specifying a final state recovers the Born rule for state $|a\rangle .{ }^{5}$ Further, when no postselection measurement

\footnotetext{
${ }^{5}$ Another way of seeing this point is to take the postselection measurement operator $P_{B=b}$ just to be the identity operator in the formulation of the ABL as $\mathcal{P}\left(c_{i} \mid a, b\right)=\frac{\left.\left|P_{B=b} P_{c=c_{i}}\right| a\right\rangle\left.\right|^{2}}{\left.\Sigma_{j}\left|P_{B=b} P_{c=c_{j}}\right| a\right\rangle\left.\right|^{2}}[4, \mathrm{p} .406]$.
} 
is made, the weak value reduces to the expectation value of the observable concerned. Thus, the standard formalism is recovered by making the final condition one that assumes no postselection. Aharonov and Rohrlich claim this choice of boundary conditions is the source of the time-asymmetry in standard QM: "by imposing an initial but not a final boundary condition, we have already sent the arrow of time flying" [3, p.137].

Next, I will consider the 'ontological' interpretation of the two-state vector.

\section{The two-time interpretation (TTI)}

Aharonov et al. introduce the Two-Time interpretation (TTI) and claim it is a deterministic, local and ontological interpretation of QM, that also solves the measurement problem $[2,9]$. In section 3.1 , I will consider the status of the backwards-evolving wavefunction within TTI and whether TTI is a hidden variable theory, before explicating their reasons for thinking it is local and deterministic. Then, in section 3.2, I will review their proposed solution to the measurement problem.

\subsection{The interpretation of $\langle\Phi|$}

This interpretation claims that there really is a backwards-evolving state vector $\langle\Phi|$ (just as much as there is a forwards-evolving state $|\Psi\rangle$ ). Therefore, at every moment in time there is a two-state vector (and therefore two Hilbert spaces) associated with the system. Ordinarily $|\uparrow\rangle_{x}$ is taken to describe the system after a projective measurement of $S_{x}$ with outcome 'up' at $t_{1}$. In TTI, $\left\langle\left.\uparrow\right|_{x}\right.$ in Figure 1 describes the system even before the postselection measurement $\left(S_{x}\right.$ at $\left.t_{1}\right)$. Further, if $\langle\Phi|$ is on equal footing with the usual state vector $|\Psi\rangle$ and an ontological reading of the quantum state is taken (as the TTI proposes ${ }^{6}$ ), then $\langle\Phi|$ has, presumably, causal power. Thus, as might be expected from a time-symmetric theory, there is retrocausality in the TTI [9, p.143]: "future and past are equally important in determining the quantum state at intermediate times, and hence equally real" [18, p.10].

This ontological interpretation of $\langle\Phi|$ in TTI goes beyond TSVF in two respects. First, the role of $\langle\Phi|$ in the weak values formula (6) and the ABL rule (4) does not require it to be assigned to the system at the earlier time $t, t_{0}<t<t_{1}$. In these formulas, one could consistently interpret it merely as the mathematical dual of the vector that describes the system later at $t_{1}$. Secondly, whilst measurements on PPS systems may, prima facie, seem contrived or artificial because

\footnotetext{
${ }^{6}$ Aharonov and Gruss deny an epistemological interpretation which "pretends only to give a set of logical rules regarding our knowledge of reality, and does not attempt to explain any underlying processes taking place" $[2, \mathrm{p} .2]$.
} 
sometimes many intermediate measurement results must be discarded ${ }^{7}$, in fact in the TTI the PPS ensemble is more fundamental than the PS ensemble. The seemingly artificial nature of performing measurements on PPS systems stems simply from our ignorance until time $t_{1}$ of the backwards-evolving wavefunction, $\langle\Phi|$. If we somehow knew $\langle\Phi|$ before $t_{1}$, we could perform the intermediate measurement only on the systems that would later pass the postselection, and we would thus not need to throw any intermediate measurement results away. Thus, the TTI takes the PPS system to be the more complete and natural description of the system even prior to the postselection measurement; systems have "an inherent final boundary condition just as all systems have an initial boundary condition" [9, p.135][26, p.11]. Thus, "in order to fully specify a system, one should not only preselect, but also postselect a certain state using a projective measurement" [18, p.10].

Now recall that if QM is a fundamental and universal theory, the quantum state $|\Psi\rangle_{S}$ with which we usually describe a system $S$, is really just a factor of a state $|\Psi\rangle_{U}=|\varphi\rangle_{S} \otimes|\Psi\rangle_{P} \otimes \ldots$ which describes the whole universe [9, 27]. Accordingly, this interpretation holds that, in addition to the initial quantum state of the universe, there is a final quantum state of the universe, of which the backwards-evolving state of $S$ is just a part. Whilst the final state of the universe is unknown, Aharonov et al. make some assumptions about its form.

These initial and final states of the universe are referred to by Aharonov and Gruss as the 'history vector' $\left|\Psi_{H I S}\right\rangle$ and the 'destiny vector' $\left\langle\Phi_{D E S}\right|$ respectively and the latter records the result of every measurement ever made [2, p.3]. As the only type of time evolution is unitary, no information is lost. As well as the two-state vector $\langle\Phi|| \Psi\rangle$, the formalism defines the two-time density matrix, which in the particular case of $\left\langle\Phi_{D E S}\right|$ and $\left|\Psi_{H I S}\right\rangle$ is,

$$
\rho=\left|\Psi_{H I S}\right\rangle\left\langle\Phi_{D E S}\right|
$$

Of course, since $\left\langle\Phi_{D E S}\right|$ and $\left|\Psi_{H I S}\right\rangle$ are, a priori, unrelated kets, $\rho$ in (7) is not hermitian - unlike usual density matrices. For more details on this novel conception of state, cf. [10, p.13], which also formulates this novel 'two-time' conception of state in the Heisenberg representation. ${ }^{8}$

I now turn to how the TTI relates to three key topics: hidden variables, nonlocality and determinism.

\footnotetext{
${ }^{7}$ Note, however, that it is possible, according to [8], to perform a weak measurement on a single particle, where all measurement results are used. My thanks to an anonymous referee for bringing this example to my attention.

${ }^{8}$ Note, however, that the formulation in [10] diverges from the TTI outlined in this paper, since the TTI is usually advertised as a local theory (cf. section 3.1.2 below), whereas [10] involves a form of non-locality that goes beyond the familiar QM-theoretic non-separability of entangled systems. This, more extreme, dynamical non-locality is manifest, for example, when a quantum particle is described as passing through one slit but exchanging a form of momentum non-locally with the other slit.
} 


\subsubsection{Is TTI a hidden variable theory?}

Is TTI a hidden variable theory like the pilot-wave theory (Bohmian mechanics)? Whilst the backwards-evolving wavefunction $\langle\Phi|$ exists at every instant of time, it is hidden from us. Only at the later time $t_{1}$ in Figures 2 and 3 are we able to discover that $\langle\Phi|=\left\langle\left.\uparrow\right|_{x}\right.$ in the interval $t_{0}<t<t_{1}$. Indeed, if we were able to access $\langle\Phi|$ prior to the postselection measurement, superluminal signalling would be possible, contradicting the no-signalling theorem (see [9, p.142]). Therefore, in the sense that it exists (according to TTI) and yet one is ignorant of it, $\langle\Phi|$ is a hidden variable. And as in other hidden variable theories, the statistical features of quantum mechanics stem from our ignorance of the hidden variables. Thus, probability in the TTI has an epistemic, rather than ontic, nature; "the probabilistic nature of quantum events can be thought of as stemming from our ignorance of the backwards-evolving state, reintroducing the classical concept of probability as a measure of our knowledge" [9, p.134].

However, TTI is not a hidden variable theory in the traditional sense. Usually a hidden variable theory claims that the system has definite values of an observable even when it is not in an eigenstate of that observable (or in the case of TTI, is neither in a forwards-evolving eigenstate nor a backwards-evolving eigenstate). But in the TTI, there are no extra value assignments in this sense.

\subsubsection{Is the TTI local?}

Aharonov and Gruss explicitly claim that the TTI is a local theory [2, p.1]. Of course, the predictions of QM violate Bell's inequalities, and so it is commonly claimed that any hidden variables must be non-local. This raises the question: how does the TTI square with Bell's theorem?

Whilst the proponents of the TTI do not explicitly relate their novel conception of state, $\langle\Phi|| \Psi\rangle$ as in equation 2 or $\rho$ as in equation (7), to the hidden variable $\lambda$ mentioned in the assumptions of Bell's theorem, I think it is clear [7] that they deny that the settings in the two wings of the EPR-Bell apparatus are independent of each other, simply because the measurement outcomes are 'recorded' in $\left\langle\left.\Phi\right|_{D E S}\right.$ and so the apparatus settings that Alice and Bob choose is encoded in $\left\langle\left.\Phi\right|_{D E S}\right.$. Thus these settings are not independent. ${ }^{9}$

More generally, setting aside the exact formulation of assumptions of a Bell theorem: the question arises how should we understand 'locality' for a theory which, like the TTI, involves retrocausality. Usually, locality requires that no event can causally influence another event outside of its future lightcone. In the TTI, the natural suggestion is therefore that locality requires that no event can

\footnotetext{
${ }^{9}$ That is, in terms of Shimony's jargon [33] of parameter independence and outcome independence: it seems to me that, taking the TTI's novel state $\rho$ to be the schematic hidden variable $\lambda$ in Shimony's conditions, TTI violates parameter independence. Nevertheless, despite this parameter dependence, we still have the no-signalling property.
} 
causally influence another event outside of its future or past lightcone.

\subsubsection{Is the TTI deterministic?}

Aharonov et al. say that the TTI is a deterministic theory, albeit in a special way. To assess this claim, recall the core idea of determinism: a theory is a set of models $T=\left\{M_{i}\right\}$, where $M_{i}$ is a possible history of the system i.e. an assignment at each time of a state to the system; and $T$ is deterministic if and only if, for all $M_{1}$ and $M_{2}$ in $T$, if $M_{1}$ and $M_{2}$ agree on the state of the system at some given time $t$, then they agree on the system's state for all future times [17].

From this definition, it is clear that whether a theory counts as deterministic will very much depend on the conception of 'state'. To avoid trivially ensuring determinism, the information encoded in a 'state' must be intrinsic to the time in question. To take an everyday example (following [17]): obviously, 'Fred was fatally wounded at noon' logically implies that Fred later died. But it does not imply that the processes leading to Fred's death were deterministic. They might well have been stochastic, and at the time of the wound, his death might even have been improbable. Thus, the apparent determinism in this case is 'cheating': it arises from encoding in a statement about noon (an attribution of a 'state' at noon, i.e. 'fatally wounded') information about the future (Fred's later demise). ${ }^{10}$

Thus, whether QM is a deterministic theory will depend how the 'state of a system' is defined and this is entwined with one's view of the measurement problem [21]. Insofar as there is no consensus on the measurement problem, there is no consensus as to whether QM is deterministic. For example, in the textbook spontaneous collapse QM theory, the state is the wavefunction and this theory is indeterministic. By contrast, in the pilot-wave theory the state of the system is described by the wavefunction and the particles' position, and consequently the theory is deterministic.

What about the TTI? Aharonov et al. claim that the theory is 'two-time deterministic': "the state of the system at any given time is completely determined by its two-state at any single time in the course of history" [9, p.142]. Thus, if the notion of state is the two-state vector $\langle\Phi|| \Psi\rangle$ of equation (2) rather than usual state vector $|\Psi\rangle$, then, they claim, the TTI is deterministic, like the pilot-wave theory. This has an epistemological construal akin to the pilot-wave theory. In the pilot-wave theory, were we to know the position of the particles in addition to the wavefunction, then predictability of measurement outcomes would be restored. Likewise, it seems that were we to know the generic backwards-evolving state $\langle\Phi|$, the TTI claims we would know the measurement outcomes.

The new terminology 'two-time determinism' is important, since the TTI flouts the standard definition given above: by defining the state as two-time state $\langle\Phi|| \Psi\rangle$,

\footnotetext{
${ }^{10}$ See [17] for discussion of natural conditions on the notion of state, in order to make determinism nontrivial.
} 
their notion, arguably, 'looks ahead' to the future in the same way that the description 'fatally wounded' does. In the last part of section 5, this claim of 'two-time determinism' is explored further.

\subsection{A review of TTI's solution to the measurement problem}

For TTI to count as an interpretation of quantum theory, it must propose a resolution to the measurement problem. The proponents of TTI describe the measurement problem as arising from the 'discrepancy' [2, p.1] between the superpositions that result from unitary evolution and single measurement outcomes. Here I review their strategy for solving this problem [2, p.4] [9, p.139] [18, p.14].

We envisage that, using the notation from Section 1, the system, apparatus and environment begin in the following state:

$$
\mid \text { Initial }\rangle=(a|\uparrow\rangle+b|\downarrow\rangle) \otimes|r e a d y\rangle \otimes|\epsilon\rangle,
$$

which unitarily evolves to

$$
(a|\uparrow\rangle \otimes|u p\rangle+b|\downarrow\rangle \otimes \mid \text { down }\rangle) \otimes|\epsilon\rangle
$$

the measuring device then interacts with the environment, encoding the interference in the environment in the usual decohering manner to

$$
\left.a|\uparrow\rangle \otimes|u p\rangle \otimes\left|\epsilon_{\text {up }}\right\rangle+b|\downarrow\rangle \otimes \mid \text { down }\right\rangle \otimes\left|\epsilon_{\text {down }}\right\rangle
$$

where $\left|\epsilon_{\text {up }}\right\rangle$ and $\left|\epsilon_{\text {down }}\right\rangle$ are orthogonal (or nearly so).

Next, the backwards-evolving final boundary condition of the entire system, expressed as a bra is taken to be

$$
\langle\text { Final }|=\langle\phi| \otimes\langle u p| \otimes\left\langle\epsilon_{u p}\right| .
$$

Crucially, the state of the measuring device is chosen to be a single member $|u p\rangle$ of the measurement basis. But the particle could have been measured in the interval between the initial time and the final time; and so it is in an arbitrary state: $|\phi\rangle=c|\uparrow\rangle+d|\downarrow\rangle$. Thus, in the intervening interval between the initial measurement to final measurement, the complete description of the composite system is given by the two-time density matrix (cf. equation (7)),

$\rho=\mid$ Initial $\rangle\left\langle\right.$ Final $|=[a|\uparrow\rangle \otimes \mid$ up $\left.\rangle \otimes| \epsilon_{\text {up }}\right\rangle+b|\downarrow\rangle \otimes \mid$ down $\left.\rangle \otimes\left|\epsilon_{\text {down }}\right\rangle\right]\left[\langle\phi| \otimes\langle u p| \otimes\left\langle\epsilon_{\text {up }}\right|\right]$

Once the environmental degrees of freedom are traced over, the reduced density 
matrix becomes (cf. equation $17[2$, p.4])

$$
\rho_{\text {reduced }}=\operatorname{Tr}_{\epsilon} \rho \approx|\uparrow\rangle \otimes|u p\rangle\langle\phi| \otimes\langle u p|
$$

Thus an effective reduction has occurred and a single measurement outcome, namely 'up', obtained.

\section{Does the TTI resolve the measurement prob- lem?}

Recall from section 1 that the criterion for solving the measurement problem was to provide 'a well-formulated theory which explains the success of the measurement algorithm' [42]. I will now argue, by considering the rationale for the choice of final measuring device state (equation 11), that the TTI does not explain the success of the measurement algorithm. At the end of this section, moreover, I will also argue that the TTI construes the concept of measurement in a suspicious way.

The choice of the final measuring device state in equation 11 is crucial to the TTI's strategy for establishing single measurement outcomes. If instead the final state were a superposition, for example,

$$
\langle\text { Final }|=\langle\phi| \otimes\left(\langle\text { up }| \otimes\left\langle\epsilon_{\text {up }}\right|+\langle\text { down }| \otimes\left\langle\epsilon_{\text {down }}\right|\right)
$$

there would be no effective reduction upon tracing over the environment. Instead the measuring device would remain in a macroscopic superposition. We would get

$$
\rho_{\text {reduced }}=\operatorname{Tr}_{\epsilon} \rho \approx|\uparrow\rangle|u p\rangle\langle\phi|\langle u p|+| \downarrow\rangle| \text { down }\rangle\langle\phi|\langle\text { down }| ;
$$

rather than as in equation (13)

$$
\rho_{\text {reduced }}=\operatorname{Tr}_{\epsilon} \rho \approx|\uparrow\rangle \otimes|u p\rangle\langle\phi| \otimes\langle u p| .
$$

Thus, a single measurement outcome would not be obtained.

A prima facie justification of the choice in equation 11 is that at the later time the measuring device was observed to be in the state 'up', rather than 'down' or a superposition. However, I submit that this justification based on experience ('we observed the measuring device pointing to "up") begs the question. Rather than explaining the success of the measurement algorithm (and thus the theory telling us why we obtain single measurement outcomes), this appeal to our experience puts the outcome 'up' into the formalism by hand - rather than specifying the dynamical processes that give rise to such outcomes.

However, Aharonov et al. give an alternative justification of the measuring device state in terms of a characterisation of all measurement devices: they say that "each measuring device has a final boundary condition equal to one of its 
possible classical states" $[2$, p.4], $[9$, p.136], $[18$, p.6]. Thus, their strategy is to appeal to all measurement devices, and thereby ultimately to cosmology. The idea is that the state of the measuring device in the final state of the universe, $\left\langle\left.\Phi\right|_{D E S}\right.$, is such that, when it is evolved backwards to $t_{1}$, its state is $\langle u p|$. This requires $\left\langle\left.\Phi\right|_{D E S}\right.$ to have a certain form. ${ }^{11}$

1. Unitary evolution usually entangles systems into superpositions which then decohere. In order that the backwards unitary evolution of a given final measuring device state in $\left\langle\left.\Phi\right|_{D E S}\right.$ does not cause it to be a superposition by $t_{1}$, it must be a 'measurement-type' state that is stable under decoherence.

2. Certain assumptions about $\left\langle\left.\Phi\right|_{D E S}\right.$ are required so as to recover the right probabilities. For instance, if $|\Psi\rangle_{D E S}$ is identical to $|\Psi\rangle_{H I S}$, then according to the ABL rule the probability of an outcome $O=o_{n}$ is proportional to $\left|\left\langle\Psi\left|P_{O=o_{n}}\right| \Psi\right\rangle\right|^{2}$, while according to the Born rule, the probability is proportional to $\left|\left\langle\Psi\left|P_{O=o_{n}}\right| \Psi\right\rangle\right|[38$, p.595]. Thus, this interpretation must stipulate that $|\Psi\rangle_{D E S} \neq|\Psi\rangle_{H I S} \cdot{ }^{12}$

Aharonov and Gruss [2] argue for the form of $\left\langle\left.\Phi\right|_{D E S}\right.$ as follows. Of an ensemble $\mathcal{E}$ of systems in the state $|\Psi\rangle=\Sigma_{i} a_{i}\left|a_{i}\right\rangle$, a random subset of systems (with a size proportional $\left|a_{1}\right|^{2}$ ) are in the state $\left|a_{1}\right\rangle$ and a random proportion $\left|a_{2}\right|^{2}$ are in the state $\left|a_{2}\right\rangle$ etc. The final states specified are elements of the measurement basis and so satisfy condition 1 above. Furthermore, $\left\langle\left.\Phi\right|_{D E S}\right.$ is stipulated not to be identical to the dual of $|\Psi\rangle_{H I S}$ but also is stipulated to recover the right empirical probabilities, thus satisfying condition 2 .

However, I submit that picking out the probabilities in this way does not constitute an explanation of the measurement algorithm. Firstly, no dynamical law has specified how certain (single) outcomes obtain. Secondly, the probabilities of single measurement outcomes are just fixed by fiat to be in accordance with the Born rule. Therefore this amounts to stipulating that the measurement algorithm works - and this is no explanation at all.

In their recent paper [9, p.136], Aharonov et al. argue that the Born rule need not be a contingent feature of the world resulting from a specific and ad hoc final state stipulation in the TTI. They claim that the Born rule, rather than any other statistical rule, follows "in the infinite $\mathrm{N}$ limit, from the compatibility of quantum mechanics with classical-like properties of macroscopic objects" [9, p.136]; (this claim is also discussed in [11]). Whilst this is of course a very interesting proposal, I submit that it is not relevant for the debate at hand since it takes as a

\footnotetext{
${ }^{11}$ Appealing to cosmology to solve the measurement problem, especially in light of the philosophical furore about the 'past hypothesis' [44, 22], may seem to be a dubious move but I will here set this aside, since as we will see shortly, the TTI proposal faces more specific problems. However, issues about $\left\langle\left.\Phi\right|_{D E S}\right.$ will recur in section 5 .

${ }^{12}$ Note this prohibition might be motivated independently of this interpretation of quantum mechanics. Gell-Mann and Hartle found that assuming $|\Psi\rangle_{D E S}=|\Psi\rangle_{H I S}$ "essentially permits no dynamics at all" [25, p.22]. Additionally, they claim that that current electromagnetic observations "may supply a probe of the final condition that is sufficiently accurate to rule out time-symmetric boundary conditions" [25, p.3], according to Davies and Twamley [46].
} 
premise the classical behaviour of macroscopic systems: but it is the occurrence of this behaviour (in particular single measurement outcomes as prescribed by the measurement algorithm, rather than macroscopic superpositions) that needs to be explained if we are to resolve the measurement problem.

Thus, I conclude that the TTI does not explain the success of the measurement algorithm. Finally, there is another, more general, problem. The TTI takes measurement to be primitive in three places, in a way that, famously, Bell [15] argued is suspicious: since measurement is presumably a physical process, it should be explained using our best physical theory, rather than taken as primitive. ${ }^{13}$

First: both the forwards and backwards-evolving wavefunctions are defined in terms of measurement outcomes at particular times. It cannot be that measurement is merely a convenient way of discovering properties of the system at $t_{0}$ and $t_{1}$ which define $|\Psi\rangle$ and $\langle\Phi|$ respectively, since according to the TTI, $\langle\Phi|$ is a property or state of the system at the earlier time $t_{0}$. Second: even if the twostate vector can be defined without reference to measurement, it was shown above that the final state of the universe has to be a privileged measurement-type state capable of determining definite states for measuring devices. Third: measurement is conceptually picked out by the fact that only at the time of measurement is it relevant to the physics that there are two vectors $|\Psi\rangle$ and $\langle\Phi|$ describing the system; they evolve independently the rest of the time.

To sum up this section: I have argued, in two stages, that the TTI proposal does not solve the measurement problem.

\section{Problems: properties and parsimony}

In this section, I outline some difficulties in the TTI about properties: problems that arise from taking $\langle\Phi|$ ontologically seriously. Then I question whether $\langle\Phi|$ should indeed be taken ontologically seriously, and ultimately I argue that we should not be committed to $\langle\Phi|$.

In $\mathrm{QM}$, we face a problem of understanding indeterminate property possession stemming from superpositions. On more traditional construals of the measurement problem, this, rather than explaining the success of the quantum algorithm, is the heart of the issue [42]. I will now argue that the existence of $\langle\Phi|$ seems to exacerbate this problem rather than solve it. The idea will be that not only do we not understand indeterminate property possession: it also seems hard to make sense in TTI of determinate property possession.

The TSVF reveals counterintuitive consequences of postselecting, such as the three box paradox (see [5],[37], [4, p.406]). In this set-up, the ABL rule tells us that

\footnotetext{
${ }^{13}$ One philosophy of science that will not be concerned about measurement being primitive is operationalism. However, operationalists (such as Fuchs and Peres [24]) will claim that no interpretation of quantum mechanics is necessary in the first place since, according to operationalism, physics is only concerned with the outcomes of experiments.
} 
an intermediate measurement will find a particle in box 1 with certainty but also in box 2 with certainty. However, the probability of finding the particle in both boxes is zero. This demonstrates the failure of the product rule for PPS systems (see [39],[4, p.408]). As the TTI adopts an ontological reading of $\langle\Phi|$, it imbues such counterintuitive consequences with ontological significance, in a manner the TSVF formalism need not be committed to. For instance, if adherents of the TTI were committed to the EPR criterion of reality ${ }^{14}$, then in the three box case, it seems they would need to ascribe contradictory properties to the system.

Even if we do not consider exotic cases like the three box paradox, there are other counterintuitive consequences. If the forwards-evolving and backwardsevolving states, $|\Psi\rangle$ and $\langle\Phi|$, are eigenstates of two (not necessarily identical) quantities, then equal treatment of them suggests that two eigenvalues should be attributed to the system. In support of this suggestion: in [10], for example, it is claimed that only eigenstates represent 'intrinsic properties'. (To be faithful to the Heisenberg representation focus of [10], this idea should be expressed as: only "a set of deterministic operators", that is "operators whose measurements do not disturb each other and have deterministic outcomes" [10, p.2] represent properties of the system.) Furthermore, adding the backwards-evolving wavefunction (or, in the language of [10], "adding a second of deterministic operators on top of the ones dictated by the initial state") amounts to "enlarging the assortment of system properties" [10, p.7]. This means that the Heisenberg indeterminacy relation does not always hold in the $\mathrm{TTI}^{15}$, in the sense that the two eigenvalues could be of incompatible observables such as position and momentum [35, p.803].

If a system is in a particular eigenstate, we traditionally say that the eigenvalue for that eigenstate represents a property of the system. But if $\langle\Phi|$ is the eigenstate $\left\langle\left.\uparrow\right|_{x}\right.$ and $\left.\mid \Psi\right\rangle$ is the eigenstate $|\uparrow\rangle_{z}$ and these reflect properties of the system, this leads the following problem, as discussed in [5]. If $S_{x}=+\frac{1}{2}$ and $S_{z}=+\frac{1}{2}$ and these eigenvalues represent properties of the system, it seems an intermediate normal i.e. strong measurement of the spin component $S_{\pi / 4}=\frac{S_{x}+S_{y}}{\sqrt{2}}$ along the $\pi / 4$ direction in the $x-z$ plane ought to yield the value $\frac{1}{\sqrt{2}}[5, \mathrm{p} .28]$. Yet this is an impermissible value; as with all components of spin, the outcome of a normal, rather than weak measurement must be either $+\frac{1}{2}$ or $-\frac{1}{2}$. Hence, assigning two eigenvalues to a given system while expecting them to reflect properties of the system which we can treat in a straightforward or intuitive way, is hard to do. ${ }^{16}$ Thus, whilst in standard

\footnotetext{
14 "If, without in any way disturbing a system, we can predict with certainty (i.e., with probability equal to unity) the value of a physical quantity, then there exists an element of reality corresponding to that quantity" [23, p.777].

${ }^{15}$ As will be discussed shortly, since the Heisenberg indeterminacy relation holds for a vector in a single Hilbert space, the result that it does not hold is perhaps incidental given that in the TTI there are two Hilbert spaces assigned to the system. My thanks to an anonymous referee for emphasising this.

${ }^{16} \mathrm{~A}$ speculative comment: perhaps we could diagnose the above problems (contradictory elements of reality, the failure of the product rule and Heisenberg uncertainty relation etc.) as resulting from an overabundance of properties one might be led to naively ascribe to a system given the TTI's conception
} 
QM there is a problem about understanding indeterminate property possession (for systems not in an eigenstate of the given quantity), the TTI faces difficulties understanding property possession even for systems that are in eigenstates.

Proponents of the TTI might reject the traditional conception of properties corresponding to eigenvalues ${ }^{17}$ and provide an alternative criterion of reality: (one such proposal is Vaidman's 'weak elements of reality' [37]). This proposal is especially relevant since the traditionally impermissible $\frac{1}{\sqrt{2}}$ value just mentioned can be 'weakly' measured on the system. Yet, if $\langle\Phi|$ only represents 'weak' properties, we may doubt that its existence is really on a par with $|\Psi\rangle$.

Given the difficulties assigning properties to a system described by $\langle\Phi|| \Psi\rangle$, we can conclude that at the very least more need to be said by proponents of the TTI. However, such a project is motivated by an ontological commitment to $\langle\Phi|$ : and I will now argue that this commitment should be doubted.

As discussed in section 3.1.3, determinism requires that the state of the system at any time (importantly, this includes the state of the system during the intermediate measurement time) is fixed by the 'state' (which in the TTI is the two-state vector) at a single time in the course of the system's history. Whilst at the time of the intermediate measurement, we cannot access $\langle\Phi|$, and thus we cannot at that time predict with certainty the measurement outcome, adding $\langle\Phi|$ is what is meant to determine the state of the system and thus, the measurement outcome.

However, generic pre- and postselection (and consequently the two-state vector) do not generally determine the outcome of the intermediate measurement. They do so (under our governing assumption that the self-Hamiltonian is zero) only in the special case where the intermediate observable is either identical to or a function of the pre or postselection observables, as shown in Figure 4. If the order of the measurements is reversed or the intermediate observable measured is incompatible with the pre and postselection observables, the outcomes are not determined. Unless the boundary conditions (PPS) are special, the outcome of the intermediate measurement is still probabilistic (indeed otherwise the ABL rule would be redundant as the probability of an outcome would be either 0 or 1 ).

This leads to two questions:

1. Since this theory is supposedly deterministic, what determines the intermediate result when $\langle\Phi|$ and $|\Psi\rangle$ do not?

2. In the case where $\langle\Phi|$ does determine the intermediate result, what determines $\langle\Phi|$ ?

According to the TTI, both questions have the same answer: there is an initial state of the universe $|\Psi\rangle_{H I S}$ and a final state of the universe $\left\langle\left.\Phi\right|_{D E S}\right.$ as discussed

of quantum reality. Indeed, Price [31] questions whether "adding an additional wavefunction 'coming from the future' to standard QM and interpreting both wavefunctions in an ontic manner"... "fail[s] to exploit the ontological efficiency possible in a retrocausal model" [31, p.79] and suggests that "a single beable may be able to play both roles".

${ }^{17}$ Indeed, a recent paper [9, p.142] suggests that this is the case. 


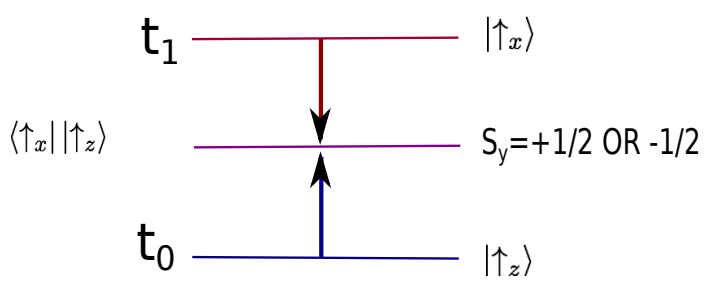

Figure 4: An intermediate measurement of $S_{y}$ in the period $t_{0}<t<t_{1}$ finds the eigenvalue $+\frac{1}{2}$ with $\operatorname{Pr}=\frac{1}{2}$ OR $-\frac{1}{2}$ with $\operatorname{Pr}=\frac{1}{2}$. This contrasts with the intermediate results in Figures 2 and 3 where the outcome was certain.

in section 3.1 and these boundary conditions determine all measurement outcomes. In section 3.1.3, two-time determinism held that the physical state at any time is determined completely by the two-state vector at any time. However, the definition of two-time determinism must be altered to 'the physical state is determined completely given the initial and final state of the universe. ${ }^{18}$ The generic twostate vector fails to determine all measurement outcomes and so the final state $\left\langle\left.\Phi\right|_{D E S}\right.$ is needed to restore determinism. ${ }^{19}$

However, this move undermines the treatment of $\langle\Phi|$ and $|\Psi\rangle$ in the two-time interpretation as ontological equals. Adding the generic backwards-evolving wavefunction $\langle\Phi|$ is not sufficient to determine the intermediate measurement outcomes: it only does so if it is special (defined by a postselection observable that is a function of the intermediate observable). Even when the backwards-evolving state does determine the intermediate measurement outcome (such as in Figures 2 and 3 ), it is not necessary to think that $\langle\Phi|$ causes it. Whilst it is consistent to think of $\langle\Phi|$ as determining the outcome in these special cases, one could equally hold that the intermediate measurement changes $|\Psi\rangle$ to $\left|\Psi^{\prime}\right\rangle$ which then determines that postselection outcome (and thus defines $\langle\Phi|$ ). Thus, attributing causal power is neither necessary nor sufficient for determining single measurement outcomes. ${ }^{20}$

Agreed, the mere availability of this alternative explanation - that the intermediate measurement determines the postselection measurement outcome rather than vice versa - does not of course compel one to accept it. Indeed, by claim-

\footnotetext{
${ }^{18}$ Clearly, this is just an outline: more needs to be said about how $\left\langle\left.\Phi\right|_{D E S}\right.$ determines measurement outcomes and about its relation to the generic backwards evolving state $\langle\Phi|$. As discussed in this section, the postselected state of the system $\langle\Phi|$ often does not suffice to determine the intermediate outcome and thus it is presumably the measuring device state in $\left\langle\left.\Phi\right|_{D E S}\right.$ that determines the intermediate measurement outcome. But this raises the question: in what sense is the measuring device sensitive to state of the system if the reading is determined by its later state as encoded in $\left\langle\left.\Phi\right|_{D E S}\right.$ ? In particular, in our familiar treatment of quantum measurement, repeating a measurement on the same system (which has a zero self-Hamiltonian) gives the same outcome again: how will this familiar feature be recovered? Here I am grateful to Emily Adlam.

${ }^{19}$ That is: determinism is restored in the two-time, and so 'looking ahead', sense discussed in section 3.1.3.

${ }^{20}$ Agreed, these considerations are themselves 'time-neutral' in the sense that one could equally argue that if we attribute causal power to $\langle\Phi|$, then attributing causal power also to the usual forwards-evolving $|\Psi\rangle$ is not necessary nor sufficient for definite measurement outcomes. (Thanks to Emily Adlam for this point). But I think a theory with only retrocausality is very counterintuitive, and so in this article, I only scrutinize attributing causal power to $\langle\Phi|$.
} 
ing that it is neither necessary nor sufficient to attribute causal power to $\langle\Phi|$ for ensuring single measurement outcomes, I am not claiming that there is no project in QM for which retrocausality may be important. But I am claiming that adding a causally efficacious backwards-evolving state is not the ingredient that needs to be added to quantum reality in order to solve the measurement problem. ${ }^{21}$

Hence, in order to ensure single measurement outcomes it is neither necessary nor (in most cases) sufficient to attribute 'causal power' to $\langle\Phi|$ and thus there is reason to doubt its existence. At the very least, it seems to have causal power to a lesser degree than the forwards-evolving state. This is reminiscent of the pilot-wave theory, where the wavefunction contributes to determining the motion of — 'kicks' the corpuscles — but the corpuscles do not 'kick' back [13], [29, p.79], [16, p.39]. Does the forwards-evolving wavefunction affect the backwards-evolving wavefunction but not vice versa? If so, then this theory might be said to violate the action-reaction principle in the same way that the pilot-wave theory has been accused of doing [13].

Thus, I believe that, even by their own lights, Aharonov et al. should reject the existence of $\langle\Phi|$ - or at least, its existence as on an equal footing with $|\Psi\rangle$. Aharonov et al. are committed to ontological parsimony [9, p.143]; their dissatisfaction with other interpretations such as the Everett and pilot-wave interpretations stems from their "requiring additional entities" and hold that "entities are not be multiplied beyond necessity" [2, p.2]. The postulated final state of the universe $\left\langle\left.\Phi\right|_{D E S}\right.$ is vital to the success of TTI; it ensures that macroscopic superpositions are not predicted and it determines measurement outcomes. The generic backwards-evolving state $\langle\Phi|$ is not so industrious: and hence ontological parsimony suggests one should not be committed to its existence.

\section{A positive proposal}

I have argued that the ontological reading of the backwards-evolving wavefunction in TTI has many undesirable features. However $\langle\Phi|$ seems highly suggestive as it arises from physical practice (postselection) and leads to novel phenomena (weak values): thus, I would now like to end by endorsing an alternative interpretation. Instead of Aharonov's proposal, I favour Vaidman's interpretation of $\langle\Phi|[38] .{ }^{22}$

If the backwards-evolving state of the measuring device is 'up' just in the case that is what is later observed, then it is an encoding of our knowledge at a later time. This epistemological interpretation is more modest; no causal power is attributed to $\langle\Phi|$. Further, $\langle\Phi|$ does not encode any properties of the system prior

\footnotetext{
${ }^{21}$ Nor is my argument an objection to Price's argument [31]. Price's argument looks at the consequences of having a time-symmetric ontology (namely that under certain assumptions, time-symmetry implies retrocausality), whereas I am concerned with whether we should have a time-symmetric ontology in the first place.

${ }^{22}$ For a full exposition of Vaidman's views on the many worlds interpretation and TSVF, see [41].
} 
to the postselection and so the difficulties around property possession and assigning two eigenvalues disappear. Moreover, this epistemological interpretation requires no assumptions about the final state of the universe. However, removing these problems comes at a price - one can no longer claim to have an interpretation of quantum mechanics. ${ }^{23}$ Yet, since I have argued that the TTI does not solve the measurement problem, I believe this is a more natural reading of $\langle\Phi|$.

\section{1 $\langle\Phi|$ and Everett}

This epistemological reading of $\langle\Phi|$ fits naturally into the Everettian framework. In the TTI's strategy to solve the measurement problem, tracing over the environment in the two-time density matrix left one measurement outcome ('up'). However, this reduction was only effective (yielding an improper rather than a proper mixture); the complete system (including the environment) is still in a superposition after the measurement. In the Everettian picture, both branches of the superposition still exist as distinct (emergent) worlds. However, $\langle u p|$ leads to the effective reduction, pointing to one branch. Thus, as suggested by Vaidman [38, p.593], the backwards-evolving state can be used to index which Everettian world you were previously in. Whereas in the TTI, an unusual weak value would be evidence for unusual final boundary conditions, in the Everettian picture it would be evidence of being in a low amplitude branch.

Indeed, the idea of a final state of the universe that cannot be known about (though it has to have a special form and contain records of all the measurements ever made) and yet causally determines the present by propagating backwards in time is, I think, no more plausible than positing a multiverse. Further, the novel phenomena such as weak values that motivated the TVSF and TTI fit well within the Everettian framework. As such, this proposal captures the desirable features of the TTI: without the difficulties outlined in this paper. However, this alternative proposal may not sit comfortably with adherents of the TTI; their philosophical motivation was to find a time-symmetric quantum theory and the Everettian branching structure is clearly asymmetric in time (see [45, ch.5]).

\section{Conclusion}

Whilst the TTI involves many interesting ideas, such as weak measurement, I believe that these ideas do not currently amount to an interpretation of QM, since — as I have argued — the two-time interpretation fails to solve the measurement problem. Since the empirical probabilities were put in by hand to the postulated final state of the universe without an underlying dynamical reason, this algorithm

\footnotetext{
${ }^{23}$ Recall that I argued in section 4 that specifying a particular outcome in accordance with our experience begs the question in the context of solving the measurement problem as it stipulates, rather than explains, single measurement outcomes.
} 
was left unexplained (section 4). Further, few would count the theory as wellformulated since the notion of measurement is primitive. In addition, I have argued that it is neither necessary nor sufficient for ensuring single measurement outcomes to attribute causal power to $\langle\Phi|$. This militates against the existence of $\langle\Phi|$. So I have advocated rejecting the TTI and its ontological reading of $\langle\Phi|$ (section 5). However, Vaidman's epistemological reading of $\langle\Phi|$ was seen to be successful, particularly within the Everettian framework (section 6).

\section{Acknowledgements}

For their help and comments on previous versions, I would like to thank three anonymous referees (especially for guidance to the burgeoning literature on the TSVF and TTI). I am indebted to Jeremy Butterfield for his extensive comments and to James Ladyman for his encouragement to work and publish on this topic. This work was funded by the Arts and Humanities Research Council.

\section{References}

[1] Aharonov, Y., Bergmann, P. G. and Lebowitz, J.L. Phys. Rev. 134, B1410 (1964).

[2] Aharonov, Y. and Gruss, E. Two-time interpretation of quantum mechanics, arXiv:quant-ph/0507269v1, (2005).

[3] Aharonov, Y. and Rohrlich, D. Quantum Paradoxes, Weinheim : Wiley-VCH, (2005).

[4] Aharonov, Y. and Vaidman, L. The two-state vector formalism: An updated review Lect. Notes Phys, 734, 399-447 (2008).

[5] Aharonov, Y., Popescu, S. and Tollaksen, J. A time-symmetric formulation of quantum mechanics Physics Today, Volume 63, Issue 11, pp. 27-33 (2010).

[6] Aharonov, Y., Albert, D. and Vaidman, L. How the Result of a Measurement of a Component of a Spin 1/2 Particle Can Turn Out to Be 100? Phys. Rev. Lett. 60, 1351-1354, (1988).

[7] Aharonov, Y., Cohen, E., Elitzur, A.C. Can a future choice affect a past measurement? Ann. Phys. 355, 258-268, (2015).

[8] Aharonov, Y., Cohen, E., Elitzur, A.C. Foundations and applications of weak quantum measurements Phys. Rev. A 89, 052105, (2014).

[9] Aharonov, Y., Cohen, E., Gruss, E., Landsberger, T. Measurement and Collapse within the Two-State Vector Formalism Quantum Stud.: Math. Found. 1, 133-146, (2014).

[10] Aharonov, Y., Landsberger, T., Cohen, E., A nonlocal ontology underlying the time-symmetric Heisenberg representation, arXiv:1510.03084v3, (2016). 
[11] Aharonov, Y., Reznik, B., How macroscopic properties dictate microscopic probabilities, Phys. Rev. A 65 052116, (2002).

[12] Albert, D. Quantum mechanics and experience, Cambridge, Mass; London: Harvard University Press, (1994).

[13] Anandan, J. and Brown, H.R. On the Reality of Space-Time Geometry and the Wavefunction, Found. Phys, Vol. 25, No. 2 (1995).

[14] Bell, J.S. Speakable and Unspeakable in Quantum Mechanics, 2nd. ed, Cambridge: Cambridge University Press, (1987).

[15] Bell, J.S. Against 'measurement' in Speakable and Unspeakable in Quantum Mechanics, 2nd. ed, Cambridge: Cambridge University Press, (1987).

[16] Bohm, D. and Hiley, B., The Undivided Universe: An Ontological Interpretation of Quantum Theory, Routledge, (1993).

[17] Butterfield, J., Determinism and indeterminism, in the Routledge Encyclopaedia of Philosophy, (2005).

[18] Cohen, E., Aharonov, Y., Quantum to Classical Transitions via Weak Measurements and Post-Selection To be published as a book chapter in "Quantum Structural Studies: Classical Emergence from the Quantum Level", R.E. Kastner, J. Jeknic-Dugic, G. Jaroszkiewicz (Eds.), World Scientific Publishing Co. (2016). arXiv:1602.05083

[19] Danan, A., Farfurnik, D., Bar-Ad, S., Vaidman, L. Asking Photons Where They Have Been PRL 111, 240402, (2013).

[20] Duck,I. M.,Stevenson, P. M., Sudarshan, E. C. G., The sense in which a "weak measurement" of a spin-particle's spin component yields a value 100 Phys. Rev. D, 40, 6, (1989).

[21] Earman, J., Determinism: what we have learned and what we still don't know, in Joseph K. Campbell (ed.), Freedom and Determinism. Cambridge Ma: Bradford Book/MIT Press 21-46, (2004).

[22] Earman, J., The past hypothesis: not even false, Studies in History and Philosophy of Science Part B: Studies in History and Philosophy of Modern Physics, 37, 3, 399-430 (2006)

[23] Einstein, A., B. Podolsky, and N. Rosen, Can Quantum-Mechanical Description of Physical Reality Be Considered Complete?, Physical Review, 47: 777780, (1935).

[24] Fuchs, C. and Peres, A. Quantum Theory needs no 'Interpretation'. Physics Today, March pp.70-71. (2000).

[25] Gell-Mann, M. and Hartle, J. Time symmetry and asymmetry in quantum mechanics and quantum cosmology in Physical Origins of Time Asymmetry, ed by J. Halliwell, J. Perez-Mercader, and W. Zurek, Cambridge University Press, Cambridge, (1994).

[26] Gruss, E. A Suggestion for a Teleological Interpretation of Quantum Mechanics, arXiv:quant-ph/0006070, (2000). 
[27] Hartle, J.B. and Hawking, S.W. The Wave Function of the Universe, Phys. Rev. D 28, 2960-2975 (1983).

[28] Hawking, S.W. The Quantum State of the Universe, Nucl. Phys. B, 239, 257276, (1984).

[29] Holland, P., The Quantum Theory of Motion, CUP, (1993).

[30] Miller, D.J. Quantum mechanics as a consistency condition on initial and final boundary conditions, Studies in History and Philosophy of Modern Physics 39, 767-781, (2008).

[31] Price, H. Does Time-Symmetry Imply Retrocausality? How the Quantum World Says "Maybe" Studies in History and Philosophy of Modern Physics, 43, 75-83, (2012).

[32] Redhead, M. Incompleteness, Nonlocality, and Realism, Oxford : Clarendon Press ; New York : Oxford University Press, (1987).

[33] Shimony, A. Events and Processes in the Quantum World in R. Penrose and C. Isham, eds., Quantum Concepts in Space and Time (Oxford: Oxford University Press), 182-203. Reprinted in Shimony 1993, 140-62. (1986)

[34] Tollaksen, J., Aharonov, Y., The Deterministic set of Operators, Quantum Interference Phenomena, and Quantum Reality, Journal of Physics: Conference Series, Vol. 196, 1. (2009).

[35] Vaidman, L, The Two-State Vector Formalism in D. Greenberger, K. Hentschel, F. Weinert, eds., Compendium of Quantum Physics: Concepts, Experiments, History and Philosophy, (Springer) (2009).

[36] Vaidman, L. Emergence of Weak Values, in Quantum Interferometry, VCH Publishers (1996), edited by F. De Martini (1996).

[37] Vaidman, L. Weak Measurement Elements of Reality Foundations of Physics, Volume 26, Issue 7, pp 895-906 (1996).

[38] Vaidman, L. Time Symmetry and the Many-Worlds Interpretation, in 'Many Worlds? Everett, Quantum theory, and Reality', (2010).

[39] L. Vaidman, Lorentz-invariant 'elements of reality' and the joint measurability of commuting observables, Phys. Rev. Lett. 70, 3369, (1993)

[40] L. Vaidman, Past of a quantum particle, Phys. Rev. A, 87, 052104, (2013).

[41] L. Vaidman, Quantum Theory and Determinism, Quantum Stud.: Math. Found. 1, 5-38, (2014). arXiv:1405.4222

[42] Wallace, D. Quantum Mechanics, Chpt. 1 in the Ashgate companion to Contemporary Philosophy of Physics, Aldershot: Ashgate, (2008).

[43] Wallace, D. Decoherence and its Role in the Modern Measurement Problem, Philosophical Transactions of the Royal Society of London, A 370 (2012).

[44] Wallace, D. Gravity, entropy, and cosmology: In search of clarity, The British Journal for the Philosophy of Science, 61, 513-540, (2010).

[45] Wallace, D. The Emergent Multiverse: Quantum Theory According to the Everett Interpretation, OUP Oxford, (2014). 
[46] Davies, P.C.W., Twamley, J., Time-symmetric cosmology and the opacity of the future lightcone Class. Quant. Grav. 10, 931, (1993). 